\section{Desigualdades na autoavaliação de saúde: uma análise para populações do Brasil e de Portugal}

\author{
Inequalities in self-rated health: an analysis of the \\ Brazilian and Portuguese populations
}

Desigualdades en la autoevaluación de salud: un
análisis para poblaciones de Brasil y de Portugal

\author{
${ }^{1}$ Centro de Ciências da \\ Saúde, Universidade Federal \\ da Paraíba, João Pessoa, \\ Brasil. \\ 2 Secretaria de Vigilância em \\ Saúde, Ministério da Saúde, \\ Brasília, Brasil. \\ ${ }^{3}$ Escola de Enfermagem, \\ Universidade Federal de \\ Minas Gerais, Belo Horizonte, \\ Brasil. \\ 4 Faculdade de Ciências \\ Médicas, Universidade \\ Estadual de Campinas, \\ Campinas, Brasil. \\ 5 Instituto de Ciências \\ Biomédicas Abel Salazar, \\ Universidade do Porto, Porto, \\ Portugal. \\ 6 Instituto de Saúde Pública, \\ Universidade do Porto, Porto, \\ Portugal. \\ 7 Departamento de \\ Epidemiologia Clínica, \\ Medicina Preditiva e Saúde \\ Pública, Universidade do \\ Porto, Porto, Portugal. \\ Correspondência \\ A. T. Carvalho \\ Departamento de Nutrição, \\ Centro de Ciências da Saúde, \\ Universidade Federal da \\ Paraíba. \\ Campus Universitário $1 \mathrm{~s} / \mathrm{n}$, \\ CCS, 2 o andar, João Pessoa, \\ PB 58059-900, Brasil. \\ alicetel@terra.com.br
}

\begin{abstract}
The aim of this study was to analyze the prevalence of poor self-rated health according to socio-demographic variables and the presence of chronic diseases in the populations of Brazil and Portugal. A total of 13,894 individuals $\geq 30$ years of age were studied in capitals in Northeast Brazil (VIGITEL 2011) and 20,579 in Portugal (4th NHI, 2005/2006). Poisson regression was used in both analyses of associations, adjusted by covariates. Net prevalence rates of poor health in men in Northeast Brazil and Portugal were $4.3 \%$ and $15.5 \%$. Prevalence ratio was 2.72 (95\%CI: 2.70-2.75) after standardization by age bracket. In women, prevalence was 8.1\% in Northeast Brazil and 25.1\% in Portugal (PR: 2.40; 95\%CI: 2.39-2.42). The variable that showed the worst inequalities in poor self-rated health was schooling, in both Brazil and Portugal. Current disease had a stronger effect on Brazilians than on Portuguese, in both men and women. Prevalence of poor self-rated health was significantly higher in Portugal in all the groups analyzed.
\end{abstract}

Self-Assessment; Health Inequalities; Health Surveys
Alice Teles de Carvalho 1

Deborah Carvalho Malta 2,3

Marilisa Berti de Azevedo Barros 4

Pedro Nuno Ferreira Pinto de Oliveira 5,6

Denisa Maria de Melo Vasques Mendonça 5,6

Henrique Barros 6,7

\section{Resumo}

Objetivou-se analisar a prevalência de saúde autoavaliada como ruim, segundo variáveis sociodemográficas e presença de doença crônica, em populações do Brasil e de Portugal. Foram estudados 13.894 indivíduos ( $\geq 30$ anos) das capitais do Nordeste do Brasil (VIGITEL 2011) e 20.579 de Portugal (4o INS 2005/2006). Utilizouse a Regressão de Poisson para as análises de associação ajustadas por covariáveis. As prevalências brutas de saúde percebida como ruim, para o Nordeste do Brasil e para Portugal, em homens, foram de 4,3\% e de 15,5\%. A razão de prevalência (RP) foi de 2,72 (IC95\%: 2,70-2,74) após a padronização por faixa etária. Para mulheres, as prevalências foram $8,1 \%$, para $o$ Brasil, e 25,1\%, para Portugal (RP: 2,40; IC95\%: 2,39-2,42). A variável que revelou desigualdades na saúde autoavaliada como ruim, em maior intensidade, foi a escolaridade, em ambas as populações. A presença de doença teve efeito maior nos brasileiros do que nos portugueses, em ambos os sexos. As prevalências de saúde autoavaliada como ruim foram significativamente mais elevadas para Portugal, em todos os agrupamentos estudados.

Autoavaliação; Desigualdades em Saúde; Inquéritos Epidemiológicos 


\section{Introdução}

A autoavaliação da saúde, obtida por meio de uma única questão, produz uma autoclassificação global da saúde do indivíduo, que é influenciada pela presença de sinais e sintomas de doenças e que reflete o impacto destas condições sobre o bem-estar físico, mental e social 1. É considerada como um indicador válido e relevante de saúde percebida, permeado por conhecimentos e crenças pessoais ${ }^{2}$. É um indicador com forte correlação com medidas objetivas de morbidade e de uso de serviços, e tem sido considerado um bom preditor da mortalidade, independentemente de fatores médicos, comportamentais e psicossociais 3 .

Uma série de análises nacionais e internacionais, realizada em países com diferentes estágios de desenvolvimento social e econômico, mostra a consistência do uso da autoavaliação da saúde como um indicador do estado de saúde de indivíduos e de populações 4,5,6,7,8,9.

No entanto, há autores 2 que, apesar de reconhecerem a relevância desse indicador, discutem e argumentam sobre a importância de uma análise, criteriosa e contextualizada, acerca das conclusões oriundas do seu uso, nas comparações entre regiões e países com grandes diferenças sociais e culturais.

Este trabalho aborda a linha de discussão da Organização Mundial da Saúde (OMS) 2, referente ao entendimento de critérios de análise que devem ser considerados para comparar os resultados obtidos com base na autoavaliação de saúde, em inquéritos populacionais de diferentes países. A análise comparativa de dados oriundos de inquéritos populacionais de países em situações de desenvolvimento socioeconômico e contextos culturais diversos contribui para o entendimento de fatores e diferenças subjetivas, que podem influenciar as respostas a uma questão global de saúde.

Em relação, especificamente, à autoavaliação de saúde na população brasileira e suas diferenças regionais, em estudo desenvolvido com dados do Sistema de Vigilância de Fatores de Risco e Proteção para Doenças Crônicas por Inquérito Telefônico (VIGITEL), realizado em 2006, em todas as capitais do Brasil, Barros et al. ${ }^{4}$ encontraram prevalências significativamente maiores de saúde autoavaliada como ruim, em indivíduos residentes em capitais do Norte e Nordeste do Brasil, regiões estas com indicadores socioeconômicos mais desfavoráveis.

Quanto a Portugal, uma análise comparativa 2 de inquéritos domiciliares sobre estado de saúde, que utilizou dados da Comissão Europeia 10 , apresenta este país como o de maior prevalência de saúde autoavaliada como ruim ou muito ruim, entre os 12 países europeus estudados. Em outra pesquisa, coordenada pela Organização para a Cooperação e Desenvolvimento Econômico (OECD) 11, em 2012, Portugal também é referido como um dos países da Europa que apresentam as prevalências mais baixas de saúde autoavaliada positivamente por sua população.

Os achados dos referidos estudos, a possibilidade de trabalhar com bases de inquéritos populacionais do Nordeste do Brasil e de Portugal e a proposta de manter o debate científico em torno do uso de inquéritos populacionais para comparações entre países nortearam o propósito deste estudo. Assim, o objetivo é analisar a prevalência de autoavaliação negativa de saúde, segundo sexo, escolaridade, faixa etária e presença de doença crônica, em populações do Brasil e de Portugal, com foco nas desigualdades identificadas dentro e entre estes países. Adicionalmente, os autores discutem os resultados na perspectiva da influência das diferenças culturais, em relação à percepção de bem-estar e de felicidade na autoavaliação de saúde.

Destaca-se que, neste trabalho, a autoavaliação de saúde é entendida como um indicador inerentemente subjetivo que está mais relacionado à percepção do que a uma avaliação objetiva sistemática 12 , ainda que fortemente correlacionado com indicadores objetivos como já mencionado.

\section{Método}

\section{Desenho e espaços do estudo}

Realizou-se um estudo seccional. Nele, foram estudados indivíduos com idade igual ou maior que trinta anos, residentes nas capitais da Região Nordeste do Brasil e em Portugal.

Fonte de dados e amostras representativas dos espaços de análise

Foram incluídas, na análise do Brasil, 13.894 pessoas residentes nas capitais da Região Nordeste (Aracaju, Fortaleza, João Pessoa, Maceió, Natal, Recife, Salvador, São Luís e Teresina), em 2011. A fonte de dados foi o VIGITEL, coordenado pela Secretaria de Vigilância em Saúde do Ministério da Saúde (SVS/MS). O VIGITEL vem sendo desenvolvido pelo Departamento de Análise de Situação de Saúde da SVS/MS desde 2006, em todas as capitais dos 26 estados brasileiros e no Distrito Federal, por meio de entrevistas telefônicas realizadas em amostras probabilísticas da população adulta, residente em domicílios servidos por linhas fixas de telefone, em cada cidade. 
Dos 18.035 indivíduos entrevistados, em 2011, pelo inquérito, $13.919(77,2 \%)$ tinham 30 anos ou mais de idade. Foram incluídos nesta análise 13.894 indivíduos que correspondem a 99,8\% dos inicialmente entrevistados nessa faixa etária e que tinham informações para as variáveis de interesse. Por se tratar de entrevista por telefone, o termo de consentimento livre e esclarecido foi substituído pelo consentimento verbal obtido por ocasião dos contatos telefônicos com os entrevistados. O VIGITEL foi aprovado pela Comissão de Ética em Pesquisa em Seres Humanos do Ministério da Saúde do Brasil.

Para Portugal, os dados de 20.579 pessoas residentes no país foram oriundos do 4 o Inquérito Nacional de Saúde (4o INS), o último realizado pelo Instituto Nacional de Saúde Dr. Ricardo Jorge (INSA), em parceria com o Instituto Nacional de Estatística (INE), no período 2005-2006. No INS são abordadas questões relacionadas à situação sociodemográfica e de saúde por meio de entrevista direta realizada com uma amostra representativa dos agregados familiares do Continente (Norte, Centro, Lisboa, Vale do Tejo, Alentejo e Algarve) e das Regiões Autônomas dos Açores e da Madeira. Dos 41.193 indivíduos entrevistados pelo 4o INS, 27.864 (67,6\%) tinham 30 anos ou mais de idade. Foram incluídos nesta análise 20.579 indivíduos, por terem informações para as variáveis de interesse, o que corresponde a 73,8\% dos inicialmente entrevistados nessa faixa etária. O inquérito foi realizado em conformidade com a Declaração de Helsinki.

Os aspectos metodológicos de amostragem e coleta de dados dos inquéritos populacionais do Brasil e de Portugal, utilizados como fontes de dados neste trabalho, estão detalhados em publicações específicas 13,14,15,16,17.

A leitura detalhada dos questionários, utilizados em cada inquérito (Brasil e Portugal) e a identificação com a análise descritiva das variáveis que os compõem, mostrou similaridade na forma de abordagem das questões de interesse deste estudo e respectivas alternativas de resposta, entre as bases dos dois inquéritos. Assim, identificou-se a possibilidade de comparabilidade nas questões objeto de análise, entre os dois espaços geográficos.

\section{Variáveis de estudo}

\section{- Variável dependente}

A variável dependente foi a autopercepção negativa do estado de saúde, que foi medida pela soma das respostas ruim ou muito ruim para o inquérito do Brasil e mau ou muito mau para o de Portugal. A forma de questionamento e as res- pectivas alternativas de respostas são similares para ambos os inquéritos. No questionário do VIGITEL, a questão está estruturada da seguinte forma: "O(a) sr(a) classificaria seu estado de saúde como?”. E as alternativas são: muito bom, bom, regular, ruim e muito ruim. No questionário do 4 o INS, a pergunta é: "De uma maneira geral, como considera o [seu] estado de saúde [do(a) senhor(a)]?”. As alternativas são muito bom, bom, razoável, mau e muito mau.

A partir desse ponto do texto, sempre que for feita referência à autopercepção de saúde ruim, deve ser considerada a soma das alternativas: ruim (mau) ou muito ruim (muito mau).

\section{- Variáveis independentes}

A prevalência de saúde autoavaliada como ruim foi analisada segundo as seguintes variáveis independentes: sexo, faixa etária, escolaridade, hipertensão arterial, diabetes e obesidade. As doenças crônicas (hipertensão e diabetes) foram consideradas presentes quando referidas pelos entrevistados, em ambos os inquéritos, como diagnosticadas por profissional de saúde e/ou quando relataram ter tido um tratamento com medicamentos específicos nas últimas duas semanas. No caso do VIGITEL, foi considerado o diagnóstico referido como realizado por médico, e para o 4o INS por enfermeiro ou médico. O índice de massa corporal $(\mathrm{IMC}=$ peso/altura 2 ) foi calculado a partir do peso (em quilogramas) e altura (em metros) referidos pelos entrevistados, e foi considerado obeso o indivíduo com IMC $\geq$ 30. A idade foi categorizada como 30-39, 40-49, 50-59, 60-69, 70-79 e 80 anos e mais. A escolaridade foi classificada baseando-se nos anos de estudos em: sem escolaridade, primário ( 1 a 4 anos), ciclo 1 (5 a 8 anos), ciclo 2 (9 a 11 anos) e superior ( $\geq 12$ anos).

\section{Análises estatísticas}

Os inquéritos têm ponderadores para correção das probabilidades de seleção das amostras e consequente extrapolação dos resultados para a população. Assim, as análises foram realizadas no módulo de Amostras Complexas do SPSS, versão 20 (IBM Corp., Armonk, Estados Unidos) e do Stata, versão 12 (StataCorp LP, College Station, Estados Unidos) que têm a opção de análise de amostras complexas e/ou permitem o uso de ponderadores.

Para investigar a associação entre saúde autoavaliada como ruim e as variáveis independentes, em cada amostra populacional, utilizou-se a regressão de Poisson (Stata). As magnitudes das associações foram medidas pelas razões de pre- 
valência (RP) com intervalo de 95\% de confiança (IC95\%).

Para a análise comparativa das prevalências de saúde autoavaliada como ruim e das prevalências de hipertensão, diabetes e obesidade, entre as capitais da Região Nordeste do Brasil e de Portugal, as estimativas foram padronizadas pelo método direto tendo a população mundial como padrão de referência. Essa etapa metodológica foi incluída, em função de diferenças na distribuição etária das amostras populacionais, na perspectiva de comparar as estimativas para os dois espaços de análise.

Ambos os inquéritos atendem aos princípios éticos contidos na Declaração de Helsinki, tendo sido aprovados por comissões nacionais específicas de cada país.

\section{Resultados}

Na Tabela 1, estão apresentadas as distribuições relativas de características demográficas (faixa etária e sexo) e de escolaridade para as amostras populacionais das capitais do Nordeste do Brasil (2011) e para Portugal (2006). A população estudada abrangeu indivíduos com trinta anos ou mais de idade. Nas duas amostras populacionais há predominância do sexo feminino (capitais do Nordeste do Brasil: 56,1\% e Portugal: 53,1\%). A amostra populacional das capitais do Nordeste do Brasil é menos envelhecida, com $65,1 \%$ dos indivíduos na faixa entre 30 e 49 anos, do que a de Portugal que apresentou $46 \%$ da população nesta faixa etária. Com $24,3 \%$ da população tendo cursado o primário ou não apresentar escolaridade formal, e 39,7\% com nível médio ou superior, a amostra populacional das capitais do Nordeste do Brasil apresentou situação de escolaridade superior à identificada para a amostra de Portugal (53,2\% com primário ou sem escolaridade). Em ambas as amostras populacionais as mulheres apresentaram situação desprivilegiada de escolaridade.

As prevalências de hipertensão foram em torno de $26,1 \%$ para os homens, em ambas as amostras, e 32,7\% (capitais do Nordeste do Brasil) e $34,8 \%$ (Portugal) para as mulheres. Para o diabetes, as estimativas foram de $7,3 \%$ para os homens e $8,9 \%$ em mulheres no Nordeste brasileiro, e em Portugal 9,2\% para os homens e 10,6\% em mulheres. A obesidade apontou cerca de 18,6\% para homens e mulheres no Brasil, e em Portugal 17\% para os homens e 19,4\% em mulheres (Tabela 2).

As prevalências brutas de saúde autoavaliada como ruim, no sexo masculino, para as capitais

Distribuição relativa de características demográficas (faixa etária e sexo) e de escolaridade das populações estudadas. Capitais do Nordeste do Brasil, 2011, e Portugal, 2006.

\begin{tabular}{|c|c|c|c|c|c|c|}
\hline \multirow[t]{2}{*}{ Variável } & \multicolumn{3}{|c|}{ Masculino (\%) } & \multicolumn{3}{|c|}{ Feminino (\%) } \\
\hline & $\begin{array}{l}\text { Brasil (capitais } \\
\text { do Nordeste) }\end{array}$ & Portugal & Valor de $p$ * & $\begin{array}{l}\text { Brasil (capitais } \\
\text { do Nordeste) }\end{array}$ & Portugal & Valor de $p$ * \\
\hline Faixa etária (anos) & & & $<0,01$ & & & $<0,01$ \\
\hline $30-39$ & 37,2 & 25,2 & & 35,5 & 22,4 & \\
\hline $40-49$ & 29,3 & 23,3 & & 28,5 & 21,4 & \\
\hline $50-59$ & 17,8 & 19,7 & & 16,6 & 18,8 & \\
\hline $60-69$ & 9,1 & 15,7 & & 11,0 & 16,3 & \\
\hline $70-79$ & 4,9 & 11,5 & & 6,0 & 13,8 & \\
\hline$\geq 80$ & 1,7 & 4,6 & & 2,4 & 7,3 & \\
\hline Escolaridade & & & $<0,01$ & & & $<0,01$ \\
\hline Superior & 14,3 & 11,4 & & 13,3 & 10,1 & \\
\hline Ciclo 2 & 25,4 & 12,1 & & 26,5 & 10,2 & \\
\hline Ciclo 1 & 38,3 & 28,6 & & 34,1 & 21,9 & \\
\hline Primário & 19,6 & 38,9 & & 22,3 & 37,8 & \\
\hline Sem escolaridade & 2,4 & 9,0 & & 3,8 & 20,0 & \\
\hline Total & 43,9 & 46,9 & $<0,01$ & 56,1 & 53,1 & $<0,01$ \\
\hline
\end{tabular}

Nota: os resultados expressam o percentual de participantes.

* Análise estatística realizada: teste qui-quadrado de independência de Pearson. 
Tabela 2

Distribuição relativa de prevalências de doenças crônicas das populações estudadas. Capitais do Nordeste do Brasil, 2011 , e Portugal, 2006.

\begin{tabular}{|c|c|c|c|c|c|c|}
\hline \multirow[t]{2}{*}{ Variável } & \multicolumn{3}{|c|}{ Masculino (\%) } & \multicolumn{3}{|c|}{ Feminino (\%) } \\
\hline & Brasil (capitais do Nordeste) & Portugal & $\mathrm{RP}$ * & Brasil (capitais do Nordeste) & Portugal & $\mathrm{RP}$ * \\
\hline \multicolumn{7}{|l|}{ Hipertensão arterial ** } \\
\hline Prevalência bruta & 26,1 & 26,4 & & 32,7 & 34,8 & \\
\hline Prevalência padronizada & 28,8 & 23,2 & 0,80 & 36,2 & 29,5 & 0,81 \\
\hline \multicolumn{7}{|l|}{ Diabetes ** } \\
\hline Prevalência bruta & 7,3 & 9,2 & & 8,9 & 10,6 & \\
\hline Prevalência padronizada & 8,6 & 7,7 & 0,89 & 10,2 & 9,0 & 0,88 \\
\hline \multicolumn{7}{|l|}{ Obesidade ** } \\
\hline Prevalência bruta & 18,6 & 17,0 & & 18,7 & 19,4 & \\
\hline Prevalência padronizada & 18,5 & 16,7 & 0,90 & 19,0 & 18,6 & 0,98 \\
\hline
\end{tabular}

IMC: índice de massa corporal; RP: razão de prevalência.

* Prevalências de hipertensão, diabetes e obesidade padronizadas por distribuição etária. Método direto. Referência: população mundial;

** Valores de referência: Nordeste do Brasil.

do Nordeste do Brasil e para Portugal, foram de $4,3 \%$ e de $15,5 \%$, respectivamente. Após a padronização, por distribuição etária, identificou-se RP de 2,72 (IC95\%: 2,70-2,74). Para o sexo feminino, as prevalências foram de $8,1 \%$ para as capitais do Nordeste brasileiro e de $25,1 \%$ para Portugal (RP: 2,40; IC95\%: 2,39-2,42). Em todos os grupos etários as prevalências de saúde autoavaliada como ruim para Portugal foram significativamente mais elevadas do que as identificadas para o Nordeste do Brasil. É perceptível, também, razões de prevalências mais elevadas nos grupos etários mais avançados e nos grupos sem diagnóstico de doenças crônicas (Tabela 3).

Em relação à escolaridade, foram identificados comportamentos diferenciados entre os sexos (Tabela 3). A amostra de homens portugueses, sem escolaridade, apresentou prevalência de saúde autoavaliada como ruim três vezes maior que a identificada para os homens, do mesmo nível de escolaridade, residentes nas capitais do Nordeste do Brasil (RP = 3,0; IC95\%: 2,98-3,02). No caso das mulheres, a desigualdade é maior no grupo do ciclo 2 de escolaridade (9 a 11 anos de estudos), que apresentou uma razão de prevalência de 1,88 (IC95\%: 1,86-1,89). Em todos os níveis de escolaridade, a desigualdade na autoavaliação de saúde, entre os residentes em Portugal e nas capitais do Nordeste do Brasil, é significativamente maior no sexo masculino.

Foram identificadas, para ambas as amostras populacionais, na análise por sexo, prevalências de saúde autoavaliada como ruim, mais elevadas para as mulheres. No Nordeste do Brasil, as prevalências brutas de 4,3\% para os homens e de $8,1 \%$ para as mulheres geraram uma razão de prevalência entre os sexos, ajustada para faixa etária, escolaridade e presença de doença crônica de 1,65 (IC95\%: 1,25-2,19). Para Portugal, as prevalências identificadas foram de $15,5 \%$ e $25,1 \%$ para homens e mulheres, respectivamente, e a razão de prevalência ajustada para as mesmas características foi de 1,29 (IC95\%: 1,20-1,39). Assim, realizou-se um modelo de análise de regressão de Poisson, estratificado por sexo, que incluiu as variáveis faixa etária, escolaridade e presença de doenças crônicas. Os resultados para o sexo masculino estão apresentados na Tabela 4 e para o sexo feminino, na Tabela 5.

Para o sexo masculino, a amostra de Portugal apresentou RP de 5,90 (IC95\%: 3,48-9,98) para a comparação entre os grupos sem escolaridade e superior com tendência de gradiente já a partir do ciclo 1 de escolaridade. Para os homens das capitais do Nordeste do Brasil, a RP entre estes grupos foi de 4,30 (IC95\%: 2,03-9,10) e as diferenças significativas foram identificadas a partir da comparação do nível primário de escolaridade com o nível superior. Para as mulheres portuguesas, RPs de 6,30 (IC95\%: 4,04-9,84) e 5,24 (IC95\%: $3,37-8,14)$ para os níveis sem escolaridade e primário, respectivamente, quando comparados com o nível superior, refletiram que a escolaridade impactou em magnitudes ainda mais elevadas na autoavaliação negativa de saúde para este grupo, quando comparado com as mulheres residentes nas capitais do Nordeste do Brasil. Para essas últimas, foram identificadas RP de 3,25 (IC95\%: 1,68-6,27) e 3,44 (IC95\%: 2,17-5,45), para os níveis sem escolaridade e primário, quando comparados à categoria de referência. 
Tabela 3

Prevalências brutas e padronizadas (distribuição etária) e razões de prevalência, de saúde autoavaliada como ruim, por sexo, faixa etária, escolaridade e presença de doença crônica. Capitais do Nordeste do Brasil, 2011 ( $n=13.894)$, e Portugal, 2006 ( $n=20.579)$.

\begin{tabular}{|c|c|c|c|c|c|c|c|c|c|c|}
\hline \multirow[t]{3}{*}{ Variável } & \multicolumn{5}{|c|}{ Masculino } & \multicolumn{5}{|c|}{ Feminino } \\
\hline & \multicolumn{2}{|c|}{ Prevalência bruta } & \multicolumn{2}{|c|}{ Prevalência padronizada } & \multirow[t]{2}{*}{ RP (IC95\%) * } & \multicolumn{2}{|c|}{ Prevalência bruta } & \multicolumn{2}{|c|}{ Prevalência padronizada } & \multirow[t]{2}{*}{$\mathrm{RP}(\mathrm{IC} 95 \%)$ * } \\
\hline & Brasil & Portugal & Brasil & Portugal & & Brasil & Portugal & Brasil & Portugal & \\
\hline \multicolumn{11}{|l|}{ Faixa etária } \\
\hline \multicolumn{11}{|l|}{ (anos) } \\
\hline 30-39 & 3,7 & 4,0 & - & - & $1,08(1,07-1,10)$ & 6,4 & 5,2 & - & - & $0,81(0,80-0,82)$ \\
\hline $40-49$ & 3,5 & 6,1 & - & - & $1,74(1,72-1,76)$ & 7,0 & 12,1 & - & - & $1,73(1,71-1,74)$ \\
\hline $50-59$ & 4,2 & 15,0 & - & - & $3,57(3,53-3,61)$ & 9,4 & 25,0 & - & - & $2,66(2,64-2,68)$ \\
\hline $60-69$ & 6,9 & 25,6 & - & - & $3,70(3,67-3,75)$ & 10,6 & 39,4 & - & - & $3,72(3,69-3,74)$ \\
\hline $70-79$ & 6,6 & 37,7 & - & - & $5,71(5,63-5,80)$ & 13,5 & 47,9 & - & - & $3,55(3,52-3,58)$ \\
\hline$\geq 80$ & 15,2 & 37,9 & - & - & $2,49(2,45-2,54)$ & 13,4 & 49,7 & - & - & $3,71(3,66-3,76)$ \\
\hline \multicolumn{11}{|l|}{ Escolaridade } \\
\hline Superior & 2,5 & 3,2 & 2,4 & 3,5 & $1,48(1,46-1,50)$ & 2,9 & 3,5 & 3,1 & 4,1 & $1,33(1,32-1,35)$ \\
\hline Ciclo 2 & 2,5 & 4,1 & 2,7 & 5,7 & $2,07(2,05-2,10)$ & 4,3 & 6,0 & 4,9 & 9,1 & $1,88(1,86-1,89)$ \\
\hline Ciclo 1 & 3,9 & 7,1 & 4,4 & 8,5 & $1,96(1,94-1,98)$ & 7,9 & 9,9 & 8,1 & 12,3 & $1,53(1,52-1,54)$ \\
\hline Primário & 7,8 & 22,8 & 8,0 & 16,8 & $2,10(2,09-2,11)$ & 14,8 & 31,2 & 14,8 & 24,4 & $1,65(1,64-1,65)$ \\
\hline Sem & 14,0 & 41,6 & 8,6 & 25,8 & $3,00(2,98-3,02)$ & 16,9 & 50,8 & 26,1 & 40,0 & $1,53(1,53-1,54)$ \\
\hline \multicolumn{11}{|l|}{ escolaridade } \\
\hline \multicolumn{11}{|l|}{ Hipertensão } \\
\hline \multicolumn{11}{|l|}{ arterial } \\
\hline Não & 3,1 & 11,4 & 3,5 & 10,8 & $3,08(3,05-3,11)$ & 5,3 & 16,9 & 5,5 & 17,2 & $3,09(3,07-3,12)$ \\
\hline Sim & 7,8 & 26,8 & 7,9 & 17,2 & $2,18(2,17-2,20)$ & 13,9 & 40,5 & 13,7 & 27,0 & $1,96(1,95-1,97)$ \\
\hline \multicolumn{11}{|l|}{ Obesidade } \\
\hline Não & 3,6 & 14,0 & 3,9 & 11,5 & $2,95(2,93-2,98)$ & 5,7 & 22,1 & 6,3 & 18,6 & $2,94(2,92-2,96)$ \\
\hline $\operatorname{Sim}$ & 5,8 & 21,8 & 6,2 & 17,4 & $2,83(2,81-2,84)$ & 13,5 & 36,3 & 14,0 & 28,1 & $2,01(2,00-2,02)$ \\
\hline \multicolumn{11}{|l|}{ Diabetes } \\
\hline Não & 3,8 & 13,2 & 4,1 & 11,2 & $2,76(2,74-2,79)$ & 7,4 & 22,4 & 7,9 & 19,2 & $2,43(2,42-2,45)$ \\
\hline $\operatorname{Sim}$ & 11,8 & 38,5 & 14,9 & 26,9 & $1,80(1,80-1,81)$ & 15,2 & 48,1 & 14,0 & 31,8 & $2,27(2,26-2,28)$ \\
\hline $\begin{array}{l}\text { Total (saúde } \\
\text { ruim) }\end{array}$ & 4,3 & 15,5 & 4,6 & 12,6 & $2,72(2,70-2,74)$ & 8,1 & 25,1 & 8,5 & 20,6 & $2,40(2,39-2,42)$ \\
\hline
\end{tabular}

IC95\%: intervalo de 95\% de confiança; RP: razão de prevalência.

* RPs estimadas com base nas prevalências padronizadas por distribuição etária, com os valores para as capitais do Nordeste do Brasil como referência.

Quanto ao impacto das doenças crônicas destacou-se, entre as estudadas, no sexo masculino, o diabetes (Tabela 4). Para os homens das capitais do Nordeste do Brasil, ser portador de diabetes resultou em RP = 2,34 (IC95\%: 1,463,73 ) e para os portugueses esta RP foi de 1,69 (IC95\%: 1,47-1,94). Em relação às mulheres (Tabela 5) tem-se para as portuguesas, em destaque, o diabetes (RP = 1,33; IC95\%: 1,22-1,44) e a hipertensão (RP = 1,31; IC95\%: 1,20-1,42), e para as mulheres das capitais do Nordeste do Brasil, a hipertensão (RP = 1,83; IC95\%: 1,31-2,54) e a obesidade (RP = 1,72; IC95\%: 1,25-2,37).

A faixa etária foi fator significativamente associado apenas para a amostra populacional de
Portugal. Nas idades mais avançadas ( $\geq 80$ anos) a prevalência de saúde autoavaliada como ruim quadruplica (RP = 4,08; IC95\%: 2,61-6,38) nos homens quando comparada com a da faixa etária entre 30 e 39 anos. Para as mulheres portuguesas a RP foi de 3,9 (IC95\%: 2,90-5,25).

\section{Discussão}

Neste estudo, o percentual da população de 30 anos ou mais, que autoavaliou a saúde como ruim, foi de $6,4 \%$ para as capitais do Nordeste do Brasil e de 20,6\% para Portugal. Após a padronização, pela distribuição etária, a prevalência 
Prevalências e razões de prevalência (ajustadas) de saúde autoavaliada como ruim, no sexo masculino, segundo variáveis sociodemográficas e presença de doenças crônicas. Capitais do Nordeste do Brasil, 2011, e Portugal, 2006.

\begin{tabular}{|c|c|c|c|c|}
\hline \multirow[t]{2}{*}{ Variável } & \multicolumn{2}{|c|}{ Brasil } & \multicolumn{2}{|c|}{ Portugal } \\
\hline & Prevalência bruta (IC95\%) & RP ajustada (IC95\%) * & Prevalência bruta (IC95\%) & RP ajustada (IC95\%) * \\
\hline \multicolumn{5}{|l|}{ Faixa etária (anos) } \\
\hline $30-39$ & $3,7(1,9-7,1)$ & 1,00 & $4,0(2,8-5,8)$ & 1,00 \\
\hline $40-49$ & $3,5(2,2-5,4)$ & $0,87(0,37-2,01)$ & $6,1(4,7-7,9)$ & $1,24(0,86-1,96)$ \\
\hline $50-59$ & $4,2(2,9-6,2)$ & $0,74(0,35-1,55)$ & $15,0(12,9-17,5)$ & $2,17(1,40-3,34)$ \\
\hline $60-69$ & $6,9(4,5-10,2)$ & $1,07(0,47-2,44)$ & $25,6(23,0-28,4)$ & $2,92(1,91-4,46)$ \\
\hline $70-79$ & $6,6(3,9-10,9)$ & $0,95(0,40-2,30)$ & $37,7(34,4-41,2)$ & $3,89(2,54-5,96)$ \\
\hline$\geq 80$ & $15,2(8,6-25,3)$ & $2,07(0,80-5,34)$ & $37,9(32,4-43,9)$ & $4,08(2,61-6,38)$ \\
\hline \multicolumn{5}{|l|}{ Escolaridade } \\
\hline Superior & $2,5(1,6-3,9)$ & 1,00 & $3,2(1,9-5,4)$ & 1,00 \\
\hline Ciclo 2 & $2,5(1,7-3,6)$ & $0,92(0,52-1,63)$ & $4,1(2,6-6,2)$ & $1,29(0,66-2,49)$ \\
\hline Ciclo 1 & $3,9(2,4-6,4)$ & $1,62(0,83-3,15)$ & $7,1(5,7-8,7)$ & $2,31(1,34-3,99)$ \\
\hline Primário & $7,8(4,8-12,4)$ & $2,10(1,12-3,94)$ & $22,8(21,0-24,7)$ & $4,28(2,55-7,18)$ \\
\hline Sem escolaridade & $14,0(8,2-23,0)$ & $4,30(2,03-9,10)$ & $41,6(37,5-45,7)$ & $5,90(3,48-9,98)$ \\
\hline \multicolumn{5}{|l|}{ Hipertensão arterial } \\
\hline Não & $3,1(2,1-4,8)$ & 1,00 & $11,4(10,4-12,5)$ & 1,00 \\
\hline Sim & $7,8(6,0-10,0)$ & $2,01(1,24-3,25)$ & $26,8(24,6-29,2)$ & $1,38(1,22-1,55)$ \\
\hline \multicolumn{5}{|l|}{ Diabetes } \\
\hline Não & $3,8(2,8-5,1)$ & 1,00 & $13,2(12,2-14,2)$ & 1,00 \\
\hline Sim & $11,8(8,2-16,6)$ & $2,34(1,46-3,73)$ & $38,5(34,3-42,9)$ & $1,69(1,47-19,4)$ \\
\hline \multicolumn{5}{|l|}{ Obesidade } \\
\hline Não & $3,6(2,6-4,9)$ & 1,00 & $14,0(13,0-15,1)$ & 1,00 \\
\hline Sim & $5,8(4,1-8,1)$ & $1,40(0,93-2,12)$ & $21,8(19,1-24,8)$ & $1,22(1,06-1,40)$ \\
\hline
\end{tabular}

IC95\%: intervalo de 95\% de confiança; RP: razão de prevalência.

* Razões de prevalências ajustadas por faixa etária, escolaridade e presença de doença crônica.

foi cerca de duas vezes e meia maior em Portugal. Esse padrão de prevalências, de saúde autoavaliada como ruim, significativamente mais elevadas para a amostra populacional de Portugal, esteve presente em todos os segmentos populacionais diferenciados segundo faixa etária, escolaridade, sexo e presença de doença crônica.

Para o entendimento desses achados é importante retornar à definição de autoavaliação de saúde entendida como um indicador de caráter valorativo, que sintetiza o estado de saúde global dos indivíduos, refletindo o seu julgamento pessoal, que é influenciado por fatores individuais, sociais, normativos e culturais 18,19. Dessa forma, a autoavaliação da saúde reflete não apenas o estado de saúde medido por indicadores objetivos da presença de doenças e incapacidades, mas também, expectativas de saúde que diferem entre indivíduos e, especialmente entre grupos populacionais inseridos em distintos contextos sociais, econômicos e culturais 2 .
Neste trabalho, a variável considerada como medida de saúde objetiva foi a presença de hipertensão, diabetes ou obesidade e as prevalências destas doenças, padronizadas por idade, foram mais elevadas para a população das capitais do Nordeste do Brasil, relativamente à de Portugal.

Quanto à situação epidemiológica, as estimativas de dois indicadores clássicos (taxa de mortalidade infantil e expectativa de vida ao nascer) da situação de saúde e condição de vida em Portugal e no Brasil, com base em instituições oficiais destes dois países, apontam para uma situação epidemiológica mais favorável a Portugal. A taxa de mortalidade infantil, por exemplo, para o ano de 2011, foi de 3,1 em Portugal 20 e de 16,43 por 1.000 nascidos vivos no Brasil 21. Já a expectativa de vida ao nascer foi de 74,20 anos para o Brasil 22 e de 79,8 para Portugal 23. Em relação ao Índice de Desenvolvimento Humano (IDH), enquanto Portugal ocupa a lista de países com Muito Alto Desenvolvimento Humano, o Brasil 
Tabela 5

Prevalências e razões de prevalência (ajustadas) de saúde autoavaliada como ruim, no sexo feminino, segundo variáveis sociodemográficas e presença de doenças crônicas. Capitais do Nordeste do Brasil, 2011, e Portugal, 2006.

\begin{tabular}{|c|c|c|c|c|}
\hline \multirow[t]{2}{*}{ Variável } & \multicolumn{2}{|c|}{ Brasil } & \multicolumn{2}{|c|}{ Portugal } \\
\hline & Prevalência bruta (IC95\%) & RP ajustada (IC95\%) * & Prevalência bruta (IC95\%) & RP ajustada (IC95\%) * \\
\hline \multicolumn{5}{|l|}{ Faixa etária (anos) } \\
\hline $30-39$ & $6,4(4,6-8,7)$ & 1,00 & $5,2(4,1-6,7)$ & 1,00 \\
\hline $40-49$ & $7,0(5,5-9,0)$ & $0,95(0,59-1,52)$ & $12,1(10,4-14,0)$ & $1,74(1,30-2,32)$ \\
\hline $50-59$ & $9,4(7,5-11,6)$ & $1,24(0,79-1,95)$ & $25,0(22,7-27,4)$ & $2,48(1,87-3,28)$ \\
\hline $60-69$ & $10,6(8,4-13,4)$ & $1,00(0,62-1,62)$ & $39,4(36,7-42,2)$ & $3,18(2,40-4,20)$ \\
\hline $70-79$ & $13,5(10,7-17,0)$ & $0,97(0,58-1,62)$ & $47,9(45,0-50,8)$ & $3,58(2,70-4,73)$ \\
\hline$\geq 80$ & $13,4(8,4-20,7)$ & $1,11(0,52-2,37)$ & $49,7(44,7-54,8)$ & $3,90(2,90-5,25)$ \\
\hline \multicolumn{5}{|l|}{ Escolaridade } \\
\hline Superior & $2,9(2,1-3,9)$ & 1,00 & $3,5(2,3-5,4)$ & 1,00 \\
\hline Ciclo 2 & $4,3(3,4-5,3)$ & $1,50(1,00-2,34)$ & $6,0(4,4-8,2)$ & $1,75(1,02-3,00)$ \\
\hline Ciclo 1 & $7,9(6,2-10,0)$ & $2,30(1,50-3,52)$ & $9,9(8,4-11,7)$ & $2,75(1,72-4,38)$ \\
\hline Primário & $14,8(12,0-18,2)$ & $3,44(2,17-5,45)$ & $31,2(29,5-33,0)$ & $5,24(3,37-8,14)$ \\
\hline Sem escolaridade & $16,9(12,2-22,9)$ & $3,25(1,68-6,27)$ & $50,8(48,2-53,4)$ & $6,30(4,04-9,84)$ \\
\hline \multicolumn{5}{|l|}{ Hipertensão arterial } \\
\hline Não & $5,3(4,3-6,6)$ & 1,00 & $16,9(15,8-18,1)$ & 1,00 \\
\hline $\operatorname{Sim}$ & $13,9(12,0-16,0)$ & $1,83(1,31-2,54)$ & $40,5(38,6-42,5)$ & $1,31(1,20-1,42)$ \\
\hline \multicolumn{5}{|l|}{ Diabetes } \\
\hline Não & $7,4(6,4-8,5)$ & 1,00 & $22,4(21,3-23,5)$ & 1,00 \\
\hline $\operatorname{Sim}$ & $15,2(12,1-18,8)$ & $1,54(1,16-2,04)$ & $48,1(44,5-51,8)$ & $1,33(1,22-1,44)$ \\
\hline \multicolumn{5}{|l|}{ Obesidade } \\
\hline Não & $5,7(4,7-6,8)$ & 1,00 & $22,1(21,0-23,2)$ & 1,00 \\
\hline Sim & $13,5(10,7-17,0)$ & $1,72(1,25-2,37)$ & $36,3(33,7-38,9)$ & $1,24(1,14-1,35)$ \\
\hline
\end{tabular}

IC95\%: intervalo de 95\% de confiança; RP: razão de prevalência.

* RPs ajustadas por faixa etária, escolaridade e presença de doença crônica.

está no grupo de Alto Desenvolvimento Humano. Essa situação de IDH, privilegiando Portugal, aparece tanto em anos mais recentes, como em 201324 , quanto em anos anteriores, por exemplo, 200625 , distanciando-se ainda mais quando se compara com as capitais do Nordeste do Brasil, espaço específico desta análise 26.

Nesse sentido, uma importante reflexão é a de que as diferenças nas prevalências de saúde autoavaliada como ruim, desfavorecendo a população de Portugal, não significam, necessariamente, que o estado geral de saúde da população das capitais do Nordeste do Brasil seja melhor, mas sim que este grupo percebe, em geral, mais positivamente, a sua situação de saúde. Nessa perspectiva, analisar comparativamente as desigualdades na prevalência de saúde autoavaliada negativamente, segundo os agrupamentos de variáveis estudadas, pode ajudar no entendimento dessas diferenças.

A prevalência de saúde autoavaliada como ruim foi significativamente mais elevada entre pessoas de menor nível de escolaridade, portadoras de doença crônica (hipertensão, diabetes ou obesidade) e do sexo feminino, tanto nas capitais do Nordeste do Brasil como em Portugal. Essas são ocorrências consistentemente relatadas em estudos que analisam a saúde autoavaliada segundo essas variáveis 4,5,27,28,29.

Entre as variáveis analisadas, o nível de escolaridade foi a que revelou maiores desigualdades na prevalência de saúde autoavaliada como ruim, tanto em homens como em mulheres e nos dois espaços de análise. Os indivíduos com níveis mais elementares (primário e sem escolaridade) de instrução apresentaram prevalências significativamente mais elevadas quando comparados aos de nível superior. Mas o achado que chama a atenção, neste estudo, é o fato de que a presença de alguma morbidade refletida por hipertensão, diabetes ou obesidade impactou, em magnitudes mais elevadas, na prevalência de saúde autoavaliada como ruim na amostra populacional das capitais da Região Nordeste do Brasil, que 
exibe razões de prevalências mais elevadas entre os grupos com e sem diagnóstico de doença, quando comparada com a amostra de Portugal. Ou seja, a presença de doença teve efeito maior nos brasileiros do que nos portugueses, em ambos os sexos, na percepção negativa da saúde. No entanto, o nível de escolaridade impactou em magnitudes mais elevadas, na prevalência de saúde autoavaliada como ruim, para Portugal.

Esses dados sugerem que as variáveis investigadas neste estudo apresentam, com a autoavaliação da saúde, relação diferenciada em cada amostra populacional analisada. Ou seja, as relações dentro de cada população estudada, entre medidas objetivas de saúde, como a presença de morbidade, e o indicador subjetivo de saúde (autopercepção de saúde ruim) são diferenciadas. Esta análise pode levantar a hipótese de que cada um dos fatores que influenciaram a proporção da população que autoavaliou a saúde como ruim, em Portugal, teve pesos diferenciados para este mesmo nível de avaliação nas capitais do Nordeste do Brasil. E, nesse caso, perceber a saúde ruim em Portugal pode não estar refletindo as mesmas dimensões de perceber a saúde ruim nas capitais nordestinas do Brasil.

Alguns fatores, como faixa-etária, sexo, níveis de renda e de escolaridade, grupos culturais e étnicos, ocupações, níveis de sociabilidade, seguridade de saúde e outros direitos, e nível global de industrialização ou desenvolvimento são reconhecidos como domínios que podem contribuir para diferentes padrões de excelência na autoavaliação de saúde 2,30. Para Sadana et al. 2, considerando padrões e expectativas diferenciadas, alguns grupos podem perceber e relatar a sua saúde como pior em relação a outro grupo, porque o seu padrão de excelência é maior do que aquele em comparação. Mas esses autores também ressaltam que tais padrões de excelência diferenciados influenciam mais nos níveis positivos (saúde boa ou muito boa) das classificações de saúde autoavaliada do que nos negativos (ruim ou muito ruim).

A valoração que os indivíduos têm da sua saúde e do seu bem-estar está relacionada com as expectativas que diferentes grupos e culturas, nos quais estes estão inseridos, têm acerca da satisfação de cada necessidade humana em relação às outras 30 . Assim, as diferenças expressas neste trabalho, com maior impacto da presença de morbidade nas prevalências de saúde autoavaliada como ruim para os brasileiros e dos diferentes agrupamentos de escolaridade, apresentando maior força de associação na autoavaliação negativa de saúde dos portugueses podem, entre outras questões, refletir a valoração diferenciada que cada um deste grupo populacional estabe- lece para cada necessidade humana, em relação às outras.

Uma linha de reflexão que pode ajudar nesse entendimento diz respeito à relação entre percepção de saúde e bem-estar subjetivo. Um estudo baseado em dados do Inquérito Social Europeu 31 , de 2004, caracterizou os níveis de bem-estar em vinte países europeus, entre os quais Portugal está incluído. Os autores utilizaram questões que refletiam aspectos emocionais do bem-estar, como sentimento de felicidade, e aspectos mais cognitivos, como a satisfação com a vida. Os resultados evidenciaram que a correlação do bem-estar e a saúde variou de acordo com o indicador utilizado, mostrando fraca correlação com indicadores objetivos como, por exemplo, dados médicos, mas apresentando fortes correlações com indicadores subjetivos da avaliação de saúde, como saúde autopercebida, sugerindo que o bem-estar influencia a percepção da saúde.

A saúde, a educação, a segurança econômica, a qualidade ambiental e a paz têm sido consideradas, em geral, como alguns dos componentes importantes do bem-estar, especialmente de caráter mais objetivo 2,32 . No aspecto subjetivo, o bem-estar tem sido concebido com base em dois componentes de autopercepção, a felicidade e a satisfação com a vida, sendo a felicidade entendida como a predominância da frequência de ocorrência de experiências emocionais positivas sobre as negativas 30,32 .

Para alguns autores 2, o limite entre a saúde e o bem-estar varia em diferentes subpopulações, contextos geográficos e culturais. Dessa forma, alguns grupos populacionais podem conceituar saúde de modo a incluir mais aspectos do bemestar subjetivo, e outros podem conceituar saúde de uma maneira a considerar aspectos mais objetivamente mensuráveis.

Nesse sentido, questões relacionadas à percepção de bem-estar, felicidade e otimismo podem contribuir para explicar as diferenças nas prevalências de saúde autoavaliada negativamente, desfavorecendo a população portuguesa. Vários estudos 30,31,33,34,35 relatam que os portugueses não têm uma visão otimista do seu bemestar. Para a população brasileira, o perfil identificado é o de uma população que se destaca por resultados positivos, em relação ao bem-estar subjetivo 34,35 .

Em relação à posição da população de Portugal entre os países europeus, quanto à percepção de bem-estar subjetivo, três estudos que utilizaram dados do Inquérito Social Europeu, um transversal, com dados de 200431 e outros dois longitudinais, um para o período de 2002 a 200830 e outro para 2002 a 2011 33, evidenciam Portugal em situação extremamente desfavorável, com 
níveis mais baixos para este indicador, avaliado com base nos componentes autopercepção de felicidade e de satisfação com a vida, mesmo quando comparado com países em situações menos favoráveis quanto a indicadores objetivos de saúde.

O Relatório Mundial sobre Felicidade de 2013 34, elaborado por especialistas a pedido da Organização das Nações Unidas, apresenta uma classificação decrescente para a felicidade no período entre 2010 e 2012, em 156 nações. Para estabelecer o ranking, foram considerados os seguintes critérios: expectativa de vida saudável, percepções de corrupção, PIB per capita, liberdade para fazer escolhas, suporte social e generosidade. O Brasil ocupa a $24^{\text {a }}$ posição nessa classificação e, Portugal, a 85a .

A publicação mais recente da pesquisa $B a$ rômetro Global de Otimismo 35, realizada em 2013 pelo Ibope Inteligência, em parceria com a Worldwide Independent Network of Market Research (WIN), que teve como objetivo medir a expectativa da população mundial para o ano de 2014, por meio dos índices de esperança e descrédito elencou, dentre sessenta e cinco países incluídos na pesquisa, o Brasil entre os dez países com a população mais otimista, tendo destaque a Região Nordeste, juntamente com as Norte e Centro-oeste. Nessa mesma publicação, Portugal encabeça a lista dos dez mais pessimistas. Um resultado, para o Brasil, que merece destaque, é a relação negativa, identificada na pesquisa, entre classe social e otimismo. Quanto mais elevada a classe social menor o índice de otimismo identificado.

Segundo Díaz et al. ${ }^{30}$, em algumas situações um elevado grau de otimismo pode representar uma espécie de mecanismo psicológico de defesa, expresso pela negação ou supressão do que tem ocorrido de mal, ocasionando uma imagem mais positiva das pessoas sobre elas mesmas e sobre as suas vidas do que seria esperado. Para os autores, no entanto, haverá momentos em que será necessário um ajustamento entre o desejo e a realidade.

A autoavaliação mais positiva da situação de saúde ou de bem-estar em grupos menos favorecidos, do ponto de vista de indicadores objetivos globais de situação de saúde e condição de vida, reafirma o impacto de indicadores subjetivos de bem-estar, que refletem a felicidade e o nível de satisfação com a vida na autoavaliação de saúde. As questões subjetivas como felicidade, otimismo e satisfação com a vida são fatores importantes e, até, fundamentais, que influenciam na autoavaliação da saúde e devem ser consideradas. Assim, em análises comparativas de autoavaliações de saúde, entre populações com graus diferenciados de bem-estar subjetivo, refletindo um maior ou menor grau de otimismo, as diferenças nas prevalências de saúde autoavaliada negativamente podem expressar, para além das diferenças na situação de saúde, aspectos psicossociais, influenciados por fundamentos culturais que impactam, diferenciadamente, cada grupo populacional.

Em relação às limitações deste estudo, no que se refere ao aspecto metodológico, está o distanciamento temporal entre os dois inquéritos analisados, considerando que para Portugal os dados de 2006 são os mais recentes oriundos do último inquérito populacional no país. Nesse sentido, os autores entendem que pode ter havido variabilidades desde então e concordam que, como refere Damián 12, no caso de avaliações que incorporam critérios subjetivos, a mudança é sem dúvida uma forma mais natural do que o estado de constância. E ainda, que qualquer mudança em uma classificação de saúde deve necessariamente ser atribuível a uma alteração nos seus determinantes, e que aspectos objetivos de saúde constituem apenas parte destes.

Outro aspecto diz respeito à abrangência territorial dos dois inquéritos: para Portugal os dados são representativos da população residente no país, já a amostra do Brasil é representativa da população residente nas capitais da Região Nordeste. Nesse sentido, a não inclusão da população da área rural na amostra do Nordeste do Brasil pode levantar discussões acerca das diferenças na situação de saúde e condição de vida, desfavorecendo a amostra do inquérito de Portugal. Um dado que pode contribuir para minimizar essa preocupação é o IDH mais elevado para Portugal referente ao período do Inquérito Nacional de Saúde de 2005/2006 (IDH = 0,904) 25 quando comparado ao IDH das capitais nordestinas para o ano de 2010, que variou de 0,721 em Maceió a 0,772 em Recife 26 .

Uma limitação a ser destacada, especificamente em relação ao estudo do VIGITEL, consiste no uso do cadastro de telefones fixos nas capitais para fins de sorteio da amostra. Nas regiões metropolitanas do Sul, Sudeste e Centro-oeste, com cobertura acima de $70 \%$, os vícios devido à exclusão de domicílios sem telefone fixo podem ser considerados desprezíveis 36 . Entretanto, as regiões Norte e Nordeste apresentaram taxas de cobertura inferiores a 70\%. Para corrigir os vícios potenciais devido à baixa cobertura de domicílios com telefone fixo, o VIGITEL utilizou o método de ponderação por célula segundo sexo, idade e escolaridade, ajustando a distribuição da amostra às características da população residente em cada capital (segundo dados de censo do Instituto Brasileiro de Geografia e Estatística - 
IBGE) e, assim, reduzindo as diferenças entre a população com e sem telefone 37 . Outros estudos comparativos entre os dados do VIGITEL e inquéritos domiciliares indicaram a importância dessas ponderações na redução dos vícios em algumas variáveis, em especial aquelas associadas à baixa renda e baixa escolaridade 36 . Os resultados deste estudo reafirmam o caráter multidimensional da saúde e contribuem para o entendimento de que a análise acerca das diferenças

\section{Resumen}

El estudio tuvo como objetivo analizar la prevalencia de salud autoevaluada como mala, según variables sociodemográficas y presencia de enfermedad crónica, en poblaciones de Brasil y Portugal. Se estudió a 13.894 individuos ( $\geq 30$ años) de capitales del Nordeste de Brasil (VIGITEL 2011) y a 20.579 de Portugal (4 INS 2005/2006). Se utilizó la regresión de Poisson para el análisis de asociación ajustado por covariables. Las prevalencias brutas de salud percibida como mala en hombres, en el Nordeste de Brasil y Portugal, fueron de un 4,3\% y de un 15,5\% respectivamente. La razón de prevalencia (RP) fue de un 2,72 (IC95\%: 2,70-2,74), tras la padronización por franja etaria. Para las mujeres, las prevalencias fueron de un 8,1\%, para Brasily un 25,1\%, para Portugal (RP: 2,40; IC95\%: 2,39-2,42). La variable que -con mayor intensidad-reveló desigualdades en la salud autoevaluada como mala fue la escolaridad, en ambas poblaciones. La presencia de enfermedad tuvo más efecto en brasileños que en portugueses, en ambos sexos. Las prevalencias de salud autoevaluada como mala fueron significativamente más elevadas para Portugal, dentro de todos los grupos estudiados.

Autoevaluación; Desigualdades en la Salud; Encuestas Epidemiológicas nas prevalências de autopercepção de saúde, entre populações com grandes diversidades socioeconômicas e culturais de desenvolvimento, deve ser feita, de forma contextualizada, incluindo outros indicadores que reflitam, objetivamente, a saúde da população e, ainda, de forma associada a variáveis subjetivas de bem-estar que incluam componentes emocionais (felicidade) e cognitivos (satisfação com a vida).

\section{Colaboradores}

A. T. Carvalho foi responsável pela concepção do projeto, delineamento do estudo, análise estatística, interpretação dos dados, revisão da literatura, estruturação e redação do texto. D. C. Malta e M. B. A. Barros participaram da concepção e delineamento do estudo, interpretação dos dados, revisão da literatura, revisão crítica e redação do texto. P. N. F. P. Oliveira participou do delineamento do estudo, análise estatística, interpretação dos dados e revisão crítica do texto. D. M. M. V. Mendonça participou do delineamento do estudo, análise estatística e revisão crítica do texto. H. Barros participou da concepção do projeto, delineamento do estudo, interpretação dos dados e revisão crítica do texto.

\section{Agradecimentos}

Agradecimentos ao Instituto Nacional de Estatística de Portugal (INE) por atender à solicitação e disponibilizar ao Prof. Henrique Barros a base de dados do Inquérito Nacional de Saúde de Portugal - 2005/2006. Ao Conselho Nacional de Desenvolvimento Científico e Tecnológico (CNPq) do Brasil pela concessão de bolsa de pós-doutorado no exterior à A. T. Carvalho (processo no 201710/2012-9). 


\section{Referências}

1. Ratner PA, Johnson JL, Jeffery B. Examining emotional, physical, social and spiritual health as determinants of self-rated health status. Am J Health Promot 1998; 12:275-82.

2. Sadana R, Mathers CD, Lopez AD, Murray CJL, Iburg KM. Comparative analyses of more than 50 household surveys on health status. In: Murray CJL, Salomon JA, Mathers CD, Lopez AD, organizadores. Summary measures of population health: concepts, ethics, measurement and applications. Geneva: World Health Organization; 2002. p. 369-86.

3. Idler EL, Benyamini Y. Self-rated health and mortality: a review of twenty-seven community studies. J Health Soc Behav 1997; 38:21-37.

4. Barros MBA, Zanchetta LM, Moura EC, Malta DC. Autoavaliação da saúde e fatores associados, Brasil, 2006. Rev Saúde Pública 2009; 43 Suppl 2:27-37.

5. Molarius A, Berglund K, Eriksson C, Lambe M, Nordström E, Eriksson HG, et al. Socioeconomic conditions, lifestyle factors, and self-rated health among men and women in Sweden. Eur J Public Health 2006; 17:125-33.

6. Alves L, Azevedo A, Barros H, Paccaud F, MarquesVidal P. Portuguese migrants in Switzerland: healthcare and health status compared to Portuguese residents. PLoS One 2013; 8:1-6.

7. Dachs JNW, Santos APR. Auto-avaliação do estado de saúde no Brasil: análise dos dados da PNAD/2003. Ciênc Saúde Coletiva 2006; 11:887-94

8. Jang Y, Kim G, Chiriboga DA. Health perception and depressive symptoms among older Korean Americans. J Cross Cult Gerontol 2006; 21:91-102.

9. Borim FSA, Barros MBA, Neri AL. Autoavaliação da saúde em idosos: pesquisa de base populacional no Município de Campinas, São Paulo, Brasil. Cad Saúde Pública 2012; 28:769-80.

10. Eurostat. Self-reported health in the European Community. Luxembourg: Eurostat; 1997. (Statistics in Focus, Population and Social Conditions).

11. Organisation for Economic Cooperation and Development. Health at a glance: Europe 2012. Paris: OECD Publishing; 2012.

12. Damián J. Re: reliability of self-rated health in us adults. Am J Epidemiol 2012; 175:857-8.

13. Secretaria de Vigilância em Saúde, Ministério da Saúde. Vigitel Brasil 2011: Vigilância de Fatores de Risco e Proteção para Doenças Crônicas por Inquérito Telefônico. Brasília: Ministério da Saúde; 2012. (Série G. Estatística e Informação em Saúde).

14. Moura EC, Morais Neto OL, Malta DC, Moura L, Silva NN, Bernal R, et al. Vigilância de Fatores de Risco para Doenças Crônicas por Inquérito Telefônico nas capitais dos 26 estados brasileiros e no Distrito Federal (2006). Rev Bras Epidemiol 2008; 11 Suppl 1:20-37.

15. Serviço de Estatísticas das Condições de Vida, Departamento de Estatísticas Sociais, Instituto Nacional de Estatística. Documento metodológico. 4o Inquérito Nacional de Saúde. Lisboa: Instituto Nacional de Estatística; 2005.

16. Instituto Nacional de Estatística; Instituto Nacional de Saúde Dr. Ricardo Jorge. Inquérito Nacional de Saúde, 2005/2006. Lisboa: Instituto Nacional de Estatística; 2009.
17. Marques-Vidal P, Dias CM. Trends in overweight and obesity in Portugal: the National Health Surveys 1995-6 and 1998-9. Obes Res 2005; 13:1141-5.

18. Borim FSA, Neri AL, Francisco PMSB, Barros MBA. Dimensões da autoavaliação de saúde em idosos. Rev Saúde Pública 2014; 48:714-722.

19. Latham K, Peek CW. Self-rated health and morbidity onset among late midlife U.S. adults. J Gerontol B Psychol Sci Soc Sci 2013; 68:107-16.

20. Fundação Francisco Manuel dos Santos. PORDATA. Bases de dados Portugal contemporâneo. http://www.pordata.pt/Portugal/Taxa+bruta+d $\mathrm{e}+$ mortalidade $+\mathrm{e}+\mathrm{taxa}+\mathrm{de}+$ mortalidade+infant il-528 (acessado em 05/Dez/2014).

21. Instituto Brasileiro de Geografia e Estatística. Brasil em síntese. http://cod.ibge.gov.br/232GH (acessado em 05/Dez/2014).

22. Instituto Brasileiro de Geografia e Estatística. Brasil em síntese. http://cod.ibge.gov.br/232i1 (acessado em 05/Dez/2014).

23. Fundação Francisco Manuel dos Santos. PORDATA. Bases de dados Portugal contemporâneo. http://www.pordata.pt/Portugal/Esperanca+de + vida+a+nascenca+total $+\mathrm{e}+$ por + sexo $+($ base + tr ienio+a+partir+de+2001)-418 (acessado em 05/ Dez/2014).

24. Programa das Nações Unidas para o Desenvolvimento. Relatório do desenvolvimento humano 2014. Sustentar o progresso humano: reduzir as vulnerabilidades e reforçar a resiliência. http:// www.pnud.org.br/arquivos/RDH2014pt.pdf (acessado em 05/Dez/2014).

25. Programa das Nações Unidas para o Desenvolvimento. Relatório do desenvolvimento humano 2009. Ultrapassar barreiras: mobilidade e desenvolvimento humanos. http://www.pnud.org.br/ HDR/arquivos/RDHglobais/hdr2009-portuguese. pdf (acessado em 05/Dez/2014).

26. Programa das Nações Unidas para o Desenvolvimento; Instituto de Pesquisa Econômica Aplicada; Fundação João Pinheiro. Ranking IDHM Municípios 2010. In: Atlas do Desenvolvimento Humano no Brasil 2013. http://www.pnud.org.br/atlas/ ranking/Ranking-IDHM-Municipios-2010.aspx (acessado em 05/Dez/2014).

27. Szwarcwald CL, Souza-Júnior PRB, Esteves MAP, Damacena GN, Viacava F. Socio-demographic determinants of self-rated health in Brazil. Cad Saúde Pública 2005; 21 Suppl 1:S54-64.

28. Asfar T, Ahmad B, Rastam S, Mulloli TP, Ward KD, Maziak W. Self-rated health and its determinants among adults in Syria: a model from the Middle East. BMC Public Health 2007; 7:177.

29. Beltrão KI, Sugahara S. Comparação de informações sobre saúde das populações brasileiras e norteamericana baseada em dados da PNAD/98 e NHIS/96. Ciênc Saúde Coletiva 2002;7:841-67.

30. Díaz R, Portela M, Neira I. Bienestar y felicidad: relación con la renta y el capital social en países europeos. Revista Galega de Economía. 2011; 20(núm. extraord.):1-29. 
31. Lima ML, Novo R. So far so good? Subjective and social well-being in Portugal and Europe. Portuguese Journal of Social Science 2006; 5:5-33.

32. Scorsolini-Comin F, Santos MA. The scientific study of happiness and health promotion: an integrative literature review. Rev Latinoam Enferm 2010; 18:472-9.

33. Pereira CR. O indivíduo a olhar para si próprio: "bom mesmo é ser um realista esperançoso". In: Ramos A, Pereira CR, Barreto J, Tavares J, Chambel MJ, Magalhães $\mathrm{P}$, et al., organizadores. 20 anos de opinião pública em Portugal e na Europa. http://www.ffms.pt/upload/docs/ebook-20anos-de-opiniao-publica-em-portugal-e-nacMh4ytA6U2QeSu3uudP8A.pdf (acessado em $\mathrm{Jul} / 2013)$.

34. Helliwell JF, Wang S. World happiness: trends, explanations and distribution. In: Helliwell J, Layard R, Sachs J, editors. World Happiness Report 2013. UN Sustainable Development Solutions Network, 2013. http://unsdsn.org/wp-content/up loads/2014/02/WorldHappinessReport2013_on line.pdf
35. IBOPE Inteligência. Voice of the People - End of the year survey. Barômetro global de otimismo. http://www.ibope.com.br/pt-br/noticias/Docu ments/win_barometro_eoy\%202013.pdf.

36. Francisco PM, Barros MB, Segri NJ, Alves MC, Cesar CL, Malta DC. Comparison of estimates for the self-reported chronic conditions among household survey and telephone survey, Campinas (SP), Brazil. Rev Bras Epidemiol 2011; 14 Suppl 1:5-15.

37. Bernal R, Silva NN. Cobertura de linhas telefônicas residenciais e vícios potenciais em estudos epidemiológicos. Rev Saúde Pública 2009; 43:421-6.

Recebido em 17/Jul/2014

Versão final reapresentada em 04/Mai/2015

Aprovado em 11/Mai/2015 